\title{
Doença Cardiovascular e Fator de risco: Percepção em Universitários
}

\author{
Enfermedad Cardiovascular y Factor de riesgo: Percepción en Universitarios \\ Cardiovascular Disease and Risk Factor: Perception in University Students
}

\author{
Isis Gabriela Santos Lemos \\ Universidade Federal do Amazonas - Amazonas - Brasil \\ Nazaré Maria de Albuquerque Hayasida \\ Universidade Federal do Amazonas - Amazonas - Brasil
}

\section{RESUMO}

A ocorrência de doenças cardiovasculares (DCV) é responsável por altas taxas de mortalidade no Brasil e no mundo. Há evidências de que o estilo de vida individual, definido como um conjunto de crenças, valores e atitudes que se refletem em nossos comportamentos apresenta um elevado impacto sobre a saúde em geral, determinando, para a maioria das pessoas, o quão doentes ou saudáveis serão a médio e longo prazo. Este estudo teve como objetivo descrever o perfil sociodemográfico, identificar FRC e analisar a percepção de risco para DCV em jovens universitários. Trata-se de pesquisa transversal, quantitativa-descritiva, com amostra de jovens adultos $(n=250)$, entre 18 a 30 anos, ambos os sexos, nos diferentes turnos e cursos de graduação em Psicologia, Enfermagem, Arquitetura e Engenharia de alimentos. Os instrumentos utilizados foram: Questionário sóciodemográfico e a Escala de Locus de Controle da Saúde. Caracterizou-se com maior frequência os FR modificáveis (sedentarismo, sobrepeso e hipercolesterolemia), e não modificáveis (herança positiva para obesidade, HAS, hipercolesterolemia e diabetes mellitus). Sugere-se prover informação e suporte para mudanças de comportamento, atendimento assistenciais e estabelecimento de objetivos como parte integrante de muitos programas educacionais.

Palavras-chave: Fatores de risco. Doença cardiovascular. Universitários.

\section{RESUMEN}

La ocurrencia de enfermedades cardiovasculares (ECV) es responsable de altas tasas de mortalidad en Brasil y en el mundo. Hay evidencias de que el estilo de vida individual, definido como un conjunto de creencias, valores y actitudes que se reflejan en nuestros comportamientos, presenta un alto impacto sobre la salud en general, determinando para la mayoría de las personas, cuán enfermos o sanos serán a medio y largo plazo. Este estudio tuvo como objetivo describir el perfil sociodemográfico, identificar FRC y analizar la percepción de riesgo para ECV en jóvenes universitarios. Se trata de una investigación transversal, cuantitativa-descriptiva, con muestra de 
jóvenes adultos $(n=250)$, entre 18 a 30 años, ambos sexos, en los diferentes turnos y cursos de graduación en Psicología, Enfermería, Arquitectura e Ingeniería de alimentos. Los instrumentos utilizados fueron: Cuestionario socio-demográfico y la Escala de Locus de Control de la Salud. Se caracterizó con mayor frecuencia los FR modificables (sedentarismo, sobrepeso e hipercolesterolemia), y no modificables (herencia positiva para obesidad, HAS, hipercolesterolemia y, diabetes mellitus). Se sugiere proveer información y soporte para cambios de comportamiento, atención asistencial y establecimiento de objetivos como parte integrante de muchos programas educativos.

Palabras clave: Factores de riesgo. Enfermedades cardiovasculares. Estudiantes universitários.

\begin{abstract}
The occurrence of cardiovascular diseases (CVD) is responsible for high mortality rates in Brazil and worldwide. There is evidence that individual life style, defined as a set of beliefs, values and attitudes reflected in our behaviors, has a high impact on overall health, determining for most people how sick or healthy they will be medium and long term. This study aimed to describe the sociodemographic profile, identify CRF and analyze the perception of risk for CVD among university students. This is a cross-sectional, quantitative-descriptive research with a sample of young adults $(n=250)$, between 18 and 30 years, both sexes, in the different shifts and undergraduate courses in Psychology, Nursing, Architecture and Food Engineering. The instruments used were: socio-demographic questionnaire and the Health Control Locus Scale. Modifiable FR (sedentary, overweight and hypercholesterolemia) were characterized more frequently (non-modifiable positive inheritance for obesity, hypertension, hypercholesterolemia and diabetes mellitus). It is suggested to provide information and support for changes in behavior, care and goal setting as an integral part of many educational programs.
\end{abstract}

Keywords: Risk factors. Cardiovascular diseases. University students.

\section{Introdução}

De acordo com a WHO (2012), no mundo há uma perspectiva de que a exposição à Fatores de Risco Cardiovascular (FRC) levará a 36 milhões de mortes prematuras até o ano de 2015, principalmente em países em desenvolvimento, que, ao contrário dos desenvolvidos, são portadores de uma população contraindo doenças, cada vez mais jovem, com frequentes complicações e mortes precoces.

Segundo
(2009), a a tendência Passos e Giatti desenvolvimento de DCV tem sido alvo de questionamentos e pesquisas. Ao avaliarem o comportamento saudável em adultos jovens no Brasil, constataram que apenas $8 \%$ encontravam-se saudáveis. Dentre os FRC avaliados, estavam inclusos prática de atividades físicas, alimentação adequada e abstinência de álcool e fumo. Gomes (2012) salientou que através da quantificação dos FRC em adultos jovens permite-se identificar o nível de suscetibilidade dos mesmos a desenvolver DCV, assim como contribuir para estratégias focadas na promoção e prevenção da saúde cardiovascular.

No Brasil, em 2009, as DCV foram responsáveis por $31,3 \%$ das mortes causadas por Doenças Crônicas Não-Transmissíveis (DCNT) (Brasil, 2006). Os fatores de risco (FR) para DCV são divididos em duas categorias: fatores de riscos modificáveis (ambientais e comportamentais) como o tabagismo, alcoolismo, colesterol sérico elevado, hipertensão arterial sistêmica, inatividade física e secundária: diabetes 
mellitus, obesidade, estresse, uso de anticoncepcional e obesidade abdominal; e fatores de risco não modificáveis (genéticos e biológicos), sendo estes, hereditariedade, sexo e idade avançada (Correia, 2010), homocisteína, variáveis sociais (suporte social e nível socioeconômico), e psicológicas (ansiedade, depressão e padrão de comportamento tipo A e estresse) (Hayasida, 2010). Estes fatores aumentam o risco de desenvolvimento da maioria das DCNT, tais como as DCV, diabetes e câncer, classificadas como maior causa de morbimortalidade na vida adulta (Barreto, Passos \& Giatti, 2009).

Para Moreira, Gomes e Santos (2010), o conceito equivocado de que os FRC e as DCV estejam presentes em fases do desenvolvimento humano mais avançadas colabora para o aumento destes em fases precoces, com evidências de aterosclerose já na idade adulta jovem compreendida entre os 20 e 40 anos. Destaca-se também que nessa fase da vida devido a busca de estabilidade no campo profissional, nas relações pessoais, dentre outros tornam-se os adultos jovens vulneráveis ao consumismo contemporâneo, que com sua influência, interfere nos comportamentos de saúde.

Conforme Barreto, Passos e Giatti (2009), países em desenvolvimento tem sua população doente cada vez mais jovem, frequentemente com complicações e mortes. Sendo que de acordo com Moreira, Gomes e Santos (2010) os comportamentos na infância influenciam a elevação de risco e de DCV na fase adulta jovem. Os autores afirmam que, a exposição a FR comportamentais, como tabagismo, alimentação inadequada e sedentarismo, frequentemente se iniciam na adolescência e se perpetuam na vida adulta. Tais fatores aumentam $\mathrm{o}$ risco de desenvolvimento de DCNT, como as cardiovasculares, diabetes e câncer, principais causas de morbimortalidade na vida adulta. Salientam que experiências e exposições, particularmente na infância e adolescência, provocam consequências a longo prazo na saúde durante a vida adulta e idosa.
Enfatizaram que a estratégia de marketing da indústria de lazer e consumo visam como público-alvo os jovens. Neste relato, os FR estão relacionados ao prazer e são disseminados na mídia, de modo a serem reforçados pela sociedade atual.

Neste contexto da fase do desenvolvimento humano, Madureira, Corseuil, Pelegrini e Petroski (2009), consideraram as situações próprias da adolescência e do adulto jovem, com significativas instabilidades biopsicossociais que somadas ao ingresso no meio universitário proporcionam novas relações sociais e a adoção de novos comportamentos, tornando os indivíduos vulneráveis às circunstâncias de risco à saúde. Por sua vez, Marcondelli, Costa e Schmtz (2008), constataram através de estudo que os acadêmicos, em sua maioria, não possuem conhecimentos científicos relevantes a respeito dos hábitos alimentares saudáveis e práticas adequadas de atividades físicas.

Mundim-Masini (2009) evidenciou que o risco é particularmente definido e percebido por indivíduos que são influenciados por vários fatores psicológicos, sociais, institucionais e culturais. Por meio de instrumentos válidos e fidedignos, esses fatores e suas relações podem ser quantificados para representar respostas das pessoas e do ambiente frente aos perigos com os quais convivem. $\mathrm{O}$ autor faz referência a questionários que sistematizam e predizem a percepção de risco, demonstrando que diferentes pessoas percebem e concebem o risco de diferentes formas.

Quando se analisa a modificação de comportamentos para a saúde, Dela Coleta (2010) sugere a importância de teorias e modelos psicológicos como excelentes auxialiares nessas ações. Propôs o construto locus de controle da saúde como uma variável que busca explicar a percepção das pessoas sobre a fonte de controle dos acontecimentos em que estão envolvidas. Deste modo, um indivíduo pode perceber-se como controlador 
destes acontecimentos ou como sendo os mesmos controlados por fatores externos a ele. A Escala de Locus de Controle da Saúde (ELCS), traduzida e validada para uso no Brasil por Dela Coleta, compreende três dimensões: Internalidade, Outros Poderosos (externalidade, atribuição a outrem) e Acaso/Sorte. Os escores fornecem, respectivamente, o grau em que a pessoa acredita em si mesma, em pessoas poderosas e no acaso, como fontes de controle da saúde (Timm, Argimon \&Wendt, 2011).

A teoria do Locus de Controle (LC) descreve que quando um esforço é apreendido pelo sujeito em alguma ação própria, mas não sendo inteiramente eventual nesta ação, ele é percebido, em nossa cultura, como consequência da sorte ou do acaso (Dela Coleta, 2010). Quando o evento é percebido dessa forma pelo indivíduo, a crença é denominada como controle externo, mas quando o indivíduo percebe que o evento é pertinente ao seu próprio comportamento ou a uma característica relativamente permanente, denominamos essa crença como sendo de controle interno (Timm, Argimon \&Wendt, 2011).

De acordo com Timm, Argimon e Wendt (2011), há estudos que denominaram os chamados controle internos como mais sensitivos a mensagens de saúde, por possuírem maior conhecimento sobre condições de saúde. Portanto, tentam melhorar seu funcionamento físico e através de esforço próprio podem ser menos suscetíveis a disfunções físicas e psicológicas, provavelmente sendo mais responsáveis por sua saúde.

Neste sentido, este estudo teve como objetivo descrever o perfil sociodemográfico, identificar FRC e analisar a percepção de risco para DCV em jovens universitários.

\section{Método}

Trata-se de um estudo transversal, descritivo-quantitativo, caracterizado por um levantamento de informações a respeito dos
FR e percepção de risco, com amostra de conveniência, constituída de 250 alunos do universo de 838 matriculados nos cursos de graduação da Universidade Federal do Amazonas (UFAM). Participaram acadêmicos entre 18 a 30 anos, de ambos os sexos, da faixa denominada "adultos jovens", conforme estratificação etária da OMS (2014). Foram entregues cartas de solicitação para participação da pesquisa aos coordenadores dos cursos de Psicologia, Enfermagem, Arquitetura e Engenharia de alimentos e agendamento para aplicação dos instrumentos junto aos responsáveis pelas Unidades de Ensino. Assim, somente foram excluídos os estudantes que apresentassem incapacidade, por alguma doença ou deficiência, de compreender ou responder às questões de forma escrita e verbal.

Os dados sociodemográficos foram coletados por meio de uma entrevista semiestruturada, elaborada para este estudo, e ELCS para avaliar suas crenças (interno ou externo). Foi aprovado pelo Comitê de Ética em Pesquisas da instituição (Protocolo CAEE no. 34983814.0.0000.5020), onde todos os participantes assinaram o Termo de Consentimento Livre e Esclarecido (TCLE) antes do início da pesquisa.

\section{Resultados}

Foi calculada uma amostragem aleatória, os dados foram inicialmente tratados por uma estatística descritiva e gráfica. Foi adotado um percentual de 50\% ( $\mathrm{p}=50 \%$ e $\mathrm{q}=50 \%$ ), haja vista que esse valor proporciona um tamanho máximo de amostra quando fixados o nível de significância $(\alpha=0,05)$ e o erro amostral relativo de $5 \%$. Após verificação da normalidade da distribuição, recorreu-se ao teste paramétrico do coeficiente de correlação de Pearson e, posteriormente à análise de regressão múltipla. Para fins de constatação sobre quais médias das dimensões estudadas são significativamente diferentes entre si, realizou-se um teste de Tukey que retornou intervalos de confianças com níveis de $95 \%$. 
Tabela 1 - Variáveis sóciodemográficas, a partir da avaliação de universitários da UFAM dos cursos de Psicologia, Enfermagem, Arquitetura e Engenharia de Alimentos ( $n=250)$.

\begin{tabular}{|c|c|c|c|c|c|c|c|c|c|c|}
\hline \multirow[b]{2}{*}{ Variável } & \multicolumn{2}{|c|}{ Psi } & \multicolumn{2}{|c|}{ Enf } & \multicolumn{2}{|c|}{ Arq } & \multicolumn{2}{|c|}{ Eng } & \multirow[b]{2}{*}{ Total } & \multirow[b]{2}{*}{$\%$} \\
\hline & $\mathrm{n}$ & $\%$ & $\mathrm{n}$ & $\%$ & $\mathrm{n}$ & $\%$ & $\mathrm{n}$ & $\%$ & & \\
\hline \multicolumn{11}{|l|}{ Sexo } \\
\hline Masculino & 16 & 6,4 & 12 & 4,8 & 25 & 10 & 18 & 7,2 & 71 & 28,4 \\
\hline Feminino & 41 & 16,4 & 73 & 29,2 & 33 & 13,2 & 32 & 12,8 & 179 & 71,6 \\
\hline Total & 57 & 22,8 & 85 & 34 & 58 & 23,2 & 50 & 20 & 250 & 100 \\
\hline \multicolumn{11}{|l|}{ Idade } \\
\hline $18-24$ & 43 & 17,2 & 58 & 23,2 & 56 & 22,4 & 48 & 19,2 & 205 & 82 \\
\hline $25-30$ & 14 & 5,6 & 27 & 10,8 & 2 & 0,8 & 2 & 0,8 & 45 & 18 \\
\hline Total & 57 & 22,8 & 85 & 34 & 58 & 23,2 & 50 & 20 & 250 & 100 \\
\hline \multicolumn{11}{|l|}{ Estado civil } \\
\hline Solteiro & 50 & 20 & 72 & 28,8 & 55 & 22 & 49 & 19,6 & 226 & 90,4 \\
\hline Casado & 6 & 2,4 & 12 & 4,8 & 3 & 1,2 & 1 & 4 & 22 & 8,8 \\
\hline Divorciado & 1 & 4 & 1 & 4 & 0 & 0 & 0 & 0 & 2 & 0,8 \\
\hline Total & 57 & 22,8 & 85 & 34 & 57 & 23,2 & 50 & 20 & 250 & 100 \\
\hline \multicolumn{11}{|l|}{ Emprego } \\
\hline Sim & 30 & 12 & 18 & 7,2 & 8 & 3,2 & 3 & 1,2 & 59 & 23,69 \\
\hline Não & 27 & 10,8 & 66 & 26,5 & 50 & 20,1 & 47 & 18,9 & 190 & 76,3 \\
\hline Total & 57 & 22,9 & 84 & 33,7 & 58 & 23,3 & 50 & 20,1 & 249 & 100 \\
\hline \multicolumn{11}{|l|}{ Mais de 1 emprego } \\
\hline Sim & 12 & 5,2 & 7 & 3 & 4 & 1,7 & 0 & 0 & 23 & 9,87 \\
\hline Não & 38 & 16,3 & 68 & 29,2 & 54 & 23,2 & 50 & 21,5 & 210 & 90,12 \\
\hline Total & 50 & 0,215 & 75 & 32,2 & 58 & 24,9 & 50 & 21,5 & 233 & 100 \\
\hline \multicolumn{11}{|l|}{ Férias do Trabalho } \\
\hline Nunca & 5 & 4,5 & 17 & 15,2 & 8 & 7,1 & 1 & 0,9 & 31 & 27,67 \\
\hline 1 vez por ano & 9 & 8 & 6 & 5,4 & 34 & 30,4 & 1 & 0,9 & 50 & 44,64 \\
\hline 2 vezes por ano & 4 & 3,6 & 6 & 5,4 & 4 & 3,6 & 0 & 0 & 14 & 12,5 \\
\hline 3 ou mais por ano & 5 & 4,5 & 6 & 5,4 & 6 & 5,4 & 0 & 0 & 17 & 15,17 \\
\hline Total & 31 & 27,7 & 50 & 44,6 & 14 & 12,5 & 17 & 15,2 & 112 & 100 \\
\hline \multicolumn{11}{|l|}{ Renda } \\
\hline$<2 \mathrm{SMM}$ & 3 & 12 & 11 & 4,4 & 0 & 0 & 5 & 2 & 19 & 7,6 \\
\hline 2 a 4 SMM & 14 & 5,6 & 10 & 4 & 12 & 22,2 & 18 & 7,2 & 54 & 21,6 \\
\hline 5 a 8 SMM & 19 & 7,6 & 47 & 18,8 & 22 & 21,2 & 16 & 6,4 & 104 & 41,6 \\
\hline$>=9 \mathrm{SMM}$ & 11 & 4,4 & 10 & 4 & 9 & 23,7 & 8 & 3,2 & 38 & 15,2 \\
\hline Não sabe & 10 & 4 & 7 & 2,8 & 15 & 4,9 & 3 & 1,2 & 35 & 14 \\
\hline Total & 57 & 22,8 & 85 & 34 & 58 & 23,2 & 50 & 20 & 250 & 100 \\
\hline \multicolumn{11}{|l|}{ Religião } \\
\hline Católica & 22 & 10,2 & 41 & 19 & 19 & 8,8 & 26 & 12 & 108 & 50 \\
\hline Protestantes e outros cristãos & 17 & 7,9 & 35 & 16,2 & 14 & 6,5 & 15 & 6,9 & 81 & 37,5 \\
\hline Outros & 7 & 3,2 & 8 & 3,7 & 11 & 5,1 & 1 & 0,5 & 27 & 12,5 \\
\hline Total & 46 & 21,3 & 84 & 38,9 & 44 & 20,4 & 42 & 19,4 & 216 & 100 \\
\hline
\end{tabular}




$\begin{array}{lcccccccccc}\text { Prática religiosa } & 26 & 10,8 & 58 & 24,2 & 27 & 11,3 & 32 & 13,3 & 143 & 59,5 \\ \text { Praticante } & 29 & 12,1 & 24 & 10 & 26 & 10,8 & 18 & 7,5 & 97 & 40,41 \\ \text { Não-praticante } & 55 & 22,9 & 82 & 34,2 & 53 & 22,1 & 50 & 20,8 & 240 & 100 \\ \text { Total } & & & & & & & & & & \end{array}$

n=frequência; \%=porcentagem

Observa-se na Tabela 1 , as principais frequências de ocorrência, houve predomínio do sexo feminino (71,6\%), faixa etária $18-24$ anos (82\%), estado civil solteiro $(90,4 \%)$, não empregados $(76,3 \%)$, que não possuem mais de 1 emprego $(90,1 \%)$, férias do trabalho $1 \mathrm{vez}$ ao ano $(44,6 \%)$, renda de 5 a 8 SMM $(41,6 \%)$, religião católica $(50 \%)$, praticante $(59,5 \%)$.
Os dados corroboram com os de Petribú, Cabral e Arruda (2009), assim como os de Barreto, Passos e Giatti (2009) que em suas amostras, obtiveram frequência maior no sexo feminino $61,6 \%$ e $50,8 \%$ no masculino, respectivamente, assim como Martins (2013) que apresentou $75 \%$ em seu estudo.

Tabela 2- Frequência do FRC Sedentarismo entre universitários da UFAM dos cursos de Psicologia, Enfermagem, Arquitetura e Engenharia de Alimentos $(n=250)$.

\begin{tabular}{lcccccc}
\hline & \multicolumn{7}{c}{ Atividade Física } \\
\cline { 2 - 7 } \multicolumn{1}{c}{ Curso } & $\mathrm{n}$ & $\%$ & $\mathrm{n}$ & $\%$ & $\mathrm{n}$ & $\%$ \\
\cline { 2 - 7 } & Pratica & Pratica & Não Pratica & Não Pratica & Total & Total \\
\hline Psicologia & 27 & 10,8 & 30 & 12,0 & 57 & 22,8 \\
Enfermagem & 40 & 16,0 & 45 & 18,0 & 85 & 34 \\
Arquitetura & 18 & 7,2 & 40 & 16,0 & 58 & 23,2 \\
Engenharia de alimentos & 18 & 7,2 & 32 & 12,8 & 50 & 20,0 \\
Total & 103 & 41,2 & 147 & 58,8 & 250 & 100 \\
\hline
\end{tabular}

$\mathrm{n}=$ frequência; \%=porcentagem

Na Tabela 2 mostra o total de alunos por curso que pratica e não pratica atividade física, sendo que houve predomínio de sedentarismo entre os alunos do curso de Enfermagem (18\%), seguido do curso de Arquitetura (16\%). É possível observar que os cursos de Psicologia (12\%) e de Engenharia de alimentos $(12,8 \%)$ possuem diminuição nas porcentagens de respostas sobre não praticar atividades esportivas.

$\mathrm{Na}$ universidade de Aveiro, em Portugal, observou-se maior sedentarismo (80\%) tanto em estudantes veteranos quanto em calouros (Brandão, Pimentel \& Cardoso, 2011). No estudo do Sistema de Vigilância por Inquérito Telefônico (VIGITEL), Iser et al. (2012) afirmou que $15,6 \%$ eram sedentários e que tende a aumentar com a idade. Em decorrência da diminuição da atividade física, o estudo aponta o aumento de excesso de peso no Brasil. Pires et al. (2013) percebeu o predomínio de sedentarismo tanto nos iniciantes quanto nos finalistas, no curso de Enfermagem. Similarmente, em pesquisa sobre estilo de vida individual de universitários, Joia (2010) expressou que a atividade física não foi revelada, e que $45 \%$ dos entrevistados não praticavam 30 minutos de exercícios físicos moderadas e/ou intensos. $\mathrm{Na}$ Universidade Federal do Piauí, Martins et al. (2010) destacou que 52\% dos acadêmicos são sedentários. Estudo sobre a prevalência de FRC ocorrido no Centro Universitário Lusíada em Santos/SP identificou 44,4\% de sedentários (Correia, Cavalcante \& Santos, 2010). No município de Juazeiro no nordeste brasileiro, Gomes et al. (2012) verificou que $57,3 \%$ dos adultos jovens são sedentários. Em uma universidade pública de Recife, são referidos $41,7 \%$ de universitários com sedentarismo (Petribú, Cabral \&Arruda, 2009). Na universidade Estadual de Goiás, Pinheiro, Fernandes e Boschetti (2009) 
observaram FRC em alunos do curso de Fisioterapia cujo sedentarismo apresentou-se como segundo FR de maior prevalência $(69,3 \%)$. Em Minas Gerais, a inatividade física foi apontada como característica do jovem em estudo sobre a prevalência de tabagismo, entre adolescentes e adultos e a influência do entorno familiar e grupo social $(34,2 \%)$ (Abreu, Souza \&Caiaffa, 2011).

Tabela 3 - Índice de Massa Corpórea (IMC) Obesidade entre universitários da UFAM dos cursos de Psicologia, Enfermagem, Arquitetura e Engenharia de Alimentos $(n=250)$.

\begin{tabular}{lcccccccccc}
\hline & \multicolumn{10}{c}{ IMC } \\
\cline { 2 - 10 } \multicolumn{1}{c}{ Curso } & $\mathrm{n}$ & $\%$ & $\mathrm{n}$ & $\%$ & $\mathrm{n}$ & $\%$ & $\mathrm{n}$ & $\%$ & $\mathrm{n}$ & $\%$ \\
\cline { 2 - 11 } & \multicolumn{1}{c}{ Baixo Peso } & \multicolumn{1}{c}{ Normal } & \multicolumn{1}{c}{ Sobrepeso } & Obeso & Total \\
\hline Psicologia & 6 & 12,2 & 28 & 57,1 & 15 & 30,6 & 2 & 0,8 & 49 & 100 \\
Enfermagem & 5 & 6,0 & 55 & 65,5 & 24 & 28,6 & 3 & 1,2 & 84 & 100 \\
Arquitetura & 5 & 8,6 & 45 & 77,6 & 8 & 13,8 & 3 & 1,2 & 58 & 100 \\
Engenharia de & & & & & & & & & & \\
alimentos & 5 & 10,2 & 28 & 57,1 & 16 & 32,7 & 3 & 1,2 & 49 & 100 \\
Total & 21 & 8,8 & 156 & 65 & 63 & 26,3 & 11 & 4,4 & 240 & 100 \\
\hline
\end{tabular}

n=frequência; \%=porcentagem

Encontram-se as frequências do IMC aferido nos alunos na Tabela 3, com dados similares quanto a obesidade, nos cursos de Enfermagem (1,2\%), Arquitetura (1,2\%) e Engenharia de alimentos $(1,2 \%)$; e sobrepeso, em Engenharia de alimentos $(32,7 \%)$ seguido do curso de Psicologia (30,6\%). Tais resultados corroboram com os achados de Marcondelli, Costa e Schmitz (2008) de 1,4\% dos participantes da área da saúde com obesidade. Em pesquisa sobre a prevalência de sobrepeso e obesidade em universitários, Simão, Nahas e Oliveira (2012) relataram sobrepeso/obesidade em $18,4 \%$ dos universitários da área de saúde e 29,7\% da área de exatas e tecnológicas, sendo que Gomes et al.(2012) referiram que 19,4\% possuem sobrepeso e obesidade. Em estudo sobre estilo de vida de jovens universitários na Espanha, Liparotti (2007) apontou que 20\% dos graduandos apresentaram excesso de peso. Petribú, Cabral e Arruda (2009) destacaram que $20,2 \%$ dos acadêmicos em uma universidade pública com obesidade. Por sua vez, Madureira et.al (2009) enfatizaram que universitários da Federal de Santa Catarina são obesos (1,85\%). Brandão, Pimentel e Cardoso (2011) realizaram estudos na universidade de Aveiro, em Portugal, destacou que 16,3\% dos alunos veteranos possuem sobrepeso em relação aos calouros com 12,5\%. Conforme Barreto, Passos e Giatti (2009), a frequência de jovens adultos obesos nas 26 capitais brasileiras e Distrito Federal foi de 7,2\%. Iser (2012) refere que 46,6\% são obesos.

Tabela 4- Frequência dos FRC Diabetes Melitus, Hipertensão Arterial Sistêmica e Hipercolesterolemia entre universitários da UFAM dos cursos de Psicologia, Enfermagem, Arquitetura e Engenharia de Alimentos $(n=250)$.

\begin{tabular}{ccccccc}
\hline & \multicolumn{2}{c}{ DM } & \multicolumn{2}{c}{ HAS } & \multicolumn{2}{c}{ Hipercolesterolemia } \\
\cline { 2 - 7 } Curso & $\mathrm{n}$ & $\%$ & $\mathrm{n}$ & $\%$ & $\mathrm{n}$ & $\%$ \\
\hline Psicologia & 2 & 0,8 & 1 & 0,4 & 10 & 4 \\
Enfermagem & 2 & 0,8 & 0 & 0 & 10 & 4 \\
Arquitetura & 0 & 0 & 0 & 0 & 7 & 2,8 \\
Engenharia de alimentos & 0 & 0 & 0 & 0 & 9 & 3,6 \\
Total & 4 & 1,6 & 1 & 0,4 & 36 & 14,4 \\
\hline
\end{tabular}

PSI UNISC, Santa Cruz do Sul, Vol. 2, n. 1,jan./jun. 2018, p.<148-155>. 
$\mathrm{Na}$ Tabela 4, destaca-se a frequência de alunos que afirmaram possuir diabetes, com predomínio nos cursos de Psicologia e Enfermagem de $0,8 \%$, sendo que não houve FR para diabetes nos cursos de Arquitetura e Engenharia de alimentos. Verificou-se que a frequência de HAS nos alunos do curso de Psicologia foi de $0,4 \%$. Não foram constatados FR de HAS nos respondentes dos cursos de Enfermagem, Arquitetura e Engenharia de alimentos. A porcentagem de hipercolesterolemia é igual nos cursos de Psicologia e Enfermagem (4\%), seguidos de 3,6\% para o curso de Engenharia de alimentos, e 2,8\% para Arquitetura.

Destaca-se, no presente estudo, a frequência de alunos com diabetes, nos cursos de Psicologia e Enfermagem de 0,8\% de universitários. Corroboram com esses dados, Gomes et al.(2012) com $0,6 \%$ de estudantes portadores de diabetes mellitus. Em universitários de uma instituição particular de Fortaleza, Veras et al. (2007) referiram que $1,2 \%$ dos estudantes são diabéticos. Porém, houve dados elevados no estudo de Petribú, Cabral e Arruda (2009) de 11,3\% dos universitários que possuem diabetes. Similarmente, na pesquisa de Barreto, Passos e Giatti (2009) foi de 10\%. Ainda, no que se refere aos FRC, em alunos da universidade de Colômbia, Feliciano-Alfonso et al. (2010) constataram 9,2\% em fase pré-diabética. Pesquisa sobre FR e proteção para DCNT em adultos no Brasil, Iser (2012) apresenta 5,8\% participantes portadores de diabetes.
Ressalta-se a frequência de HAS entre universitários da UFAM, compreendendo o curso de Psicologia $(0,4 \%)$. Por sua vez, Gomes et al. (2012) encontraram 1,1\% de adultos jovens hipertensos, em Juazeiro, no nordeste brasileiro. Em Assunção no Paraguai, Campagnoli, Gonzalez e Cruz (2012) verificaram que $3 \%$ encontrava-se com HAS e que o consumo de sal e valores de pressão arterial foram significativamente elevados em adultos jovens. Assim como, em estudantes de Enfermagem, Alves e Marques (2009) destacaram 3,6\%. Pinheiro, Fernandes e Boschetti (2009) observaram o percentual de FRC com menor prevalência de HAS, sendo $6,5 \%$ de sua amostra identificada como portadora desse tipo de DCNT. No estudo de Barreto, Passos e Giatti (2009), verificou-se $6 \%$ de portadores de HAS entre as 26 capitais brasileiras e Distrito Federal.

Sobre hipercolesterolemia, os achados de Petribú, Arruda e Cabral (2009) não corrobora com os resultados desse estudo, pois revelam $62,7 \%$ com colesterol acima do recomendado. No estudo sobre FRC em uma população portuguesa, um dos resultados obtidos foi a prevalência de hipercolesterolemia entre os alunos de Ciências da Saúde (20,2\%) (Brandão et al., 2008). Pesquisa de Mariano et al. (2013), identificou $19,51 \%$ portadores de hipercolesterolemia. Em Fortaleza, a análise do perfil lipídico de universitários realizada por Freitas et al. (2013) revelaram 9,7\% de alunos com valores de colesterol elevado.

Tabela 5 - Frequência do FRC Herança Familiar para Diabetes entre universitários da UFAM dos cursos de Psicologia, Enfermagem, Arquitetura e Engenharia de Alimentos ( $n=250)$.

\begin{tabular}{|c|c|c|c|c|c|c|}
\hline \multicolumn{7}{|c|}{ Família- Diabetes } \\
\hline \multirow{2}{*}{ Curso } & \multicolumn{2}{|c|}{ Sim } & \multicolumn{2}{|c|}{ Não } & \multicolumn{2}{|c|}{ Total } \\
\hline & $\mathrm{n}$ & $\%$ & $\mathrm{~N}$ & $\%$ & $\mathrm{n}$ & $\%$ \\
\hline Psicologia & 23 & 9,2 & 34 & 13,6 & 57 & 22,8 \\
\hline Enfermagem & 49 & 19,6 & 36 & 14,4 & 85 & 34 \\
\hline Arquitetura & 25 & 10 & 33 & 13,2 & 58 & 23,2 \\
\hline Engenharia de alimentos & 23 & 9,2 & 27 & 10,8 & 50 & 20 \\
\hline Total & 120 & 48 & 130 & 52 & 250 & 100 \\
\hline
\end{tabular}

PSI UNISC, Santa Cruz do Sul, Vol. 2, n. 1,jan./jun. 2018, p.<143-155>. 
n=frequência; \%=porcentagem

$\mathrm{Na}$ Tabela 5, destaca-se a maior frequência de histórico familiar para diabetes no curso de Enfermagem (19,6\%), sendo que $48 \%$ dos analisados possuem hereditariedade para essa doença. Esse resultado apoia a pesquisa de Gomes et al. (2012) cuja prevalência foi de $46,7 \%$ de história familiar para diabetes. Assim como Correia,
Cavalcante e Santos (2010) observaram $54,55 \%$ com antecedentes familiares. Entretanto, os achados de Petribú, Cabral e Arruda (2009) correspondentes a 11,2\% de herança positiva para diabetes não corroboram com os resultados da presente pesquisa. Assim como Veras et al. (2007) apresentou valor mais elevado $(72,1 \%)$.

Tabela 6 - Frequência do FRC Herança Familiar para hipertensão arterial (HAS) entre universitários da UFAM dos cursos de Psicologia, Enfermagem, Arquitetura e Engenharia de Alimentos $(n=250)$.

\begin{tabular}{cccccccc}
\hline \multicolumn{1}{c}{ Família- HAS } \\
\cline { 2 - 7 } Curso & \multicolumn{3}{c}{ Sim } & \multicolumn{2}{c}{ Não } & \multicolumn{2}{c}{ Total } \\
\cline { 2 - 7 } & $\mathrm{n}$ & $\%$ & $\mathrm{n}$ & $\%$ & $\mathrm{n}$ & $\%$ \\
\hline Psicologia & 29 & 11,6 & 28 & 11,2 & 57 & 22,8 \\
Enfermagem & 62 & 24,8 & 23 & 9,2 & 85 & 34 \\
Arquitetura & 30 & 12 & 28 & 11,2 & 58 & 23,2 \\
Engenharia de alimentos & 25 & 10 & 25 & 10 & 50 & 20 \\
Total & 146 & 58,4 & 104 & 41,6 & 250 & 100 \\
\hline
\end{tabular}

n=frequência; \%=porcentagem

Na Tabela 6, houve predomínio de hereditariedade para HAS no curso de Enfermagem (24,8\%), com destaque de 58,4\% nos cursos analisados. Esses achados confirmaram os de Gomes et al.(2012) com prevalência de $68,7 \%$ de herança genética para HAS advindos do pai ou da mãe, similarmente aos dados de Correia, Cavalcante e Santos (2010) com 63,64\% de universitários com herança positiva.

Petribú, Cabral e Arruda (2009) referiram a prevalência de $35,2 \%$ de universitários com antecedentes familiares, por sua vez, Veras et al. (2007) apontaram $76,2 \%$ desse FRC.

Tabela 7 - Frequência do FRC Herança Familiar para Obesidade entre universitários da UFAM dos cursos de Psicologia, Enfermagem, Arquitetura e Engenharia de Alimentos $(n=250)$.

Família- Obesidade

\begin{tabular}{|c|c|c|c|c|c|c|}
\hline \multirow[b]{3}{*}{ Curso } & \multirow{2}{*}{\multicolumn{2}{|c|}{$\mathrm{Sim}$}} & \multirow{2}{*}{\multicolumn{2}{|c|}{ Não }} & & \\
\hline & & & & & \multicolumn{2}{|c|}{ Total } \\
\hline & $\mathrm{n}$ & $\%$ & $\mathrm{n}$ & $\%$ & $\mathrm{n}$ & $\%$ \\
\hline Psicologia & 12 & 4,8 & 45 & 18 & 57 & 22,8 \\
\hline Enfermagem & 30 & 12 & 55 & 22 & 85 & 34 \\
\hline Arquitetura & 15 & 6 & 43 & 17,2 & 58 & 23,2 \\
\hline Engenharia de alimentos & 13 & 5,2 & 37 & 14,8 & 50 & 20 \\
\hline Total & 70 & 28 & 180 & 72 & 250 & 100 \\
\hline
\end{tabular}

n=frequência; \%=porcentagem

Na Tabela 7, houve predomínio de histórico familiar para obesidade, constatada no curso de Enfermagem (12\%), compreendendo-se que $28 \%$ dos universitários

PSI UNISC, Santa Cruz do Sul, Vol. 2, n. 1,jan./jun. 2018, p.<143-155>. 
relataram ter FR para essa doença. O estudo de Petribú, Arruda e Cabral (2009) respalda esses achados, posto que $20 \%$ dos universitários de sua pesquisa são portadores do referido FR.
Em contrapartida, Veras et al.(2007) identificaram $5,9 \%$ de acadêmicos com antecedentes familiares para obesidade.

Tabela 8- Frequência do FRC Herança Familiar para hipercolesterolemia entre universitários da UFAM dos cursos de Psicologia, Enfermagem, Arquitetura e Engenharia de Alimentos $(n=250)$.

\begin{tabular}{|c|c|c|c|c|c|c|}
\hline \multicolumn{7}{|c|}{ Família- Hipercolesterolemia } \\
\hline \multirow{2}{*}{ Curso } & \multicolumn{2}{|c|}{ Sim } & \multicolumn{2}{|c|}{ Não } & \multicolumn{2}{|c|}{ Total } \\
\hline & $\mathrm{n}$ & $\%$ & $\mathrm{n}$ & $\%$ & $\mathrm{n}$ & $\%$ \\
\hline Psicologia & 21 & 8,4 & 36 & 14,4 & 57 & 22,8 \\
\hline Enfermagem & 45 & 18 & 40 & 16 & 85 & 34 \\
\hline Arquitetura & 32 & 12,8 & 26 & 10,4 & 58 & 23,2 \\
\hline Engenharia de alimentos & 24 & 9,6 & 26 & 10,4 & 50 & 20 \\
\hline Total & 122 & 48,8 & 128 & 51,2 & 250 & 100 \\
\hline
\end{tabular}

Quanto aos dados da Tabela 8, observa-se maior frequência de alunos com histórico familiar para hipercolesterolemia, no curso de Enfermagem (18\%), sendo que 48,8\% possuem familiares acometidos por essa doença. Esses dados corroboram com os de Gomes et al. (2012) que apresentaram 34,8\%. Por sua vez, Veras et al. (2007) evidenciaram dados elevados $(60,5 \%)$ de universitários com o mesmo FR.

\section{Análise dos dados relativos à Escala de Locus de Controle da Saúde (ELCS)}

Os dados quantitativos relativos ao indicador da percepção pessoal sobre quem ou o quê controla a saúde dos universitários são expostos na Tabela 9. Inicialmente, apresentase a estatística descritiva, com informações sobre médias e desvios padrão da população estudada.

Tabela 9 - Locus de Controle da Saúde nos cursos de Psicologia, Enfermagem, Arquitetura e Engenharia de alimentos $(n=250)$.

\begin{tabular}{|c|c|c|c|}
\hline \multicolumn{4}{|c|}{ Psicologia } \\
\hline Dimensões & $\mathrm{n}$ & $\mathrm{M}$ & DP \\
\hline Internalidade & 57 & 21,73 & 2,97 \\
\hline Outros Poderosos & 57 & 17,14 & 3,55 \\
\hline Externalidade & 57 & 12,56 & 3,99 \\
\hline \multicolumn{4}{|c|}{ Enfermagem } \\
\hline Dimensões & $\mathrm{n}$ & M & DP \\
\hline Internalidade & 85 & 22,25 & 3,58 \\
\hline Outros Poderosos & 85 & 17,3 & 4,21 \\
\hline Externalidade & 85 & 11,94 & 3,9 \\
\hline \multicolumn{4}{|c|}{ Arquitetura } \\
\hline Dimensões & $\mathrm{n}$ & M & Desvio Padrão \\
\hline Internalidade & 58 & 21,96 & 4,33 \\
\hline Outros Poderosos & 58 & 16,25 & 3,78 \\
\hline Externalidade & 58 & 12,46 & 3,93 \\
\hline Curso & Iaria & & \\
\hline
\end{tabular}

PSI UNISC, Santa Cruz do Sul, Vol. 2, n. 1,jan./jun. 2018, p.<151-155>. 


\begin{tabular}{cccc} 
Dimensões & $\mathrm{n}$ & $\mathrm{M}$ & $\mathrm{DP}$ \\
\hline Internalidade & 50 & 22,4 & 3,9 \\
Outros Poderosos & 50 & 18,52 & 4,6 \\
Externalidade & 50 & 12,78 & 4,14 \\
\hline
\end{tabular}

$\mathrm{n}=$ frequência; $\mathrm{M}=$ média; $\mathrm{DP}=$ desvio padrão

No curso de Psicologia houve maior frequência da dimensão Internalidade $(\mathrm{M}=$ 21,73 e DP $=52,97$ ), que indica o grau que acreditam que eles próprios controlam sua saúde. Houve diferença entre os escores obtidos nas dimensões para o curso de Psicologia $[F(1,108)=104,16 ; p<0,01]$. No curso de Enfermagem, todas as dimensões diferiram entre si, sendo que houve predomínio da dimensão Internalidade $(\mathrm{M}=$ 22,25 e DP $=17,31$ ), sobre como os alunos pensam e fazem sobre sua saúde, a atribuição é relacionada em sua maioria a si mesmos. Sendo que somente a dimensão Externalidade não apresentou distribuição normal, portanto foi submetida ao teste não paramétrico (Friedman). Houve diferença entre as dimensões para o curso de Enfermagem (Friedman $\mathrm{p}<0,01$ ).

No curso de Arquitetura todas as dimensões foram diferentes entre si e a dimensão Internalidade teve maior frequência $(\mathrm{M}=21,96$ e $\mathrm{DP}=4,33)$. Houve diferença entre as dimensões no curso de Arquitetura [F $(1,106)=74,12 ; \mathrm{p}<0,01]$.

No curso de Engenharia de alimentos todas as dimensões mostraram diferença entre si, sendo a Internalidade predominante $(\mathrm{M}=22,4$ e $\mathrm{DP}=3,9)$. Houve diferença entre as dimensões para o curso de Engenharia de alimentos $[\mathrm{F}(1,95)=75,69 ; \mathrm{p}<0,01]$.

\section{Considerações finais}

O conteúdo de maior relevância desta análise está nos FRC modificáveis, por se inserir neste âmbito o estilo de vida. Todavia, os FR não modificáveis foram mencionados devido ao sinergismo entre eles que são cruciais na manifestação da DCV. Em relação à presença de FRC, houve maior frequência dos FR modificáveis, como o sedentarismo, sobrepeso e hipercolesterolemia, e os não modificáveis (herança positiva para obesidade, HAS, hipercolesterolemia e diabetes mellitus).

No que se refere ao FR não modificável (herança positiva para obesidade, HAS, hipercolesterolemia e diabetes mellitus) foi representativa na maioria dos universitários da amostra. É fundamental salientar que a HAS contribui para o seguimento de complicações vasculares e de cardiopatia. Estas informações devem nortear a criação de um programa preventivo em relação aos riscos aqui estudados a respeito do sobrepeso, ser direcionadas à dieta, apontando para a necessidade de reeducação alimentar e não de dietas curtas, sendo a dieta uma conduta a ser implementada, pois funciona como um dos principais fatores dentro dos fatores modificáveis.

Sobre o LC, os dados revelam que em relação ao que pensam e fazem sobre a sua própria saúde, a maioria atribui a responsabilidade a si mesmo. Para que o autocuidado tenha sucesso é necessário perceber as próprias necessidades, ou seja, indagar-se sobre o que é realmente preciso ter no estilo de vida para manter a saúde. A percepção do indivíduo sobre um problema a ser enfrentado, em seu ritmo natural, é um fator importante que influencia na reação para a busca de melhorias. Assim, há possibilidade de harmonizar a saúde com o viver do cotidiano. Algumas pessoas que acreditam que o seu locus é interno baseiam-se nos resultados, bons ou ruins, que ocorrem em função de algo que fizeram ou que eventualmente deixaram de fazer. Portanto, culpam-se pelos fracassos e assumem atitudes pelo sucesso (Guerra, 2010). O controle interno tem sido importante para os cuidados e adoção de comportamentos de saúde (redução do peso excessivo, controle de HAS), sendo 
uma variável significativa para o desenvolvimento de programas de intervenção na promoção da saúde e na prevenção da doença (Diretrizes da Sociedade Brasileira de Cardiologia, 2009-2014).

A detecção precoce da presença de FR no jovem adulto possibilita o planejamento e a implementação de programas intervencionistas e preventivos direcionados à redução da probabilidade de manifestação das DCV no futuro. Sugere-se o desenvolvimento de um instrumento específico para avaliar a percepção de risco cardiovascular, além de psicoeducação nas universidades através de eventos científicos, oficinas e seminários que possibilitem o acesso ao conhecimento dos FRC pelos universitários, visando capacitá-los como multiplicadores desses conhecimentos.

O presente estudo revela algumas limitações, no tocante a reflexão: mesmo se fornecêssemos aos universitários as informações sobre os cuidados e as escolhas mais adequadas e saudáveis, aquelas que lhes parecessem melhor, ainda assim a efetividade na mudança comportamental não seria garantida. Consideremos o comportamento uma variável multifatorial que implica em motivação, recursos financeiros, dentre outros.

\section{Referências}

Abreu, M.N.S; Souza, C.F; Caiaffa, W.T. (2011). Tabagismo entre adolescentes e adultos jovens de Belo Horizonte, Minas Gerais, Brasil: influência do entorno familiar e grupo social. Caderno de Saúde Pública, Rio de Janeiro, 27 (5), maio, p. 935-943.

Alves, A.A.S.; Marques, I.R. (2009). Fatores relacionados ao risco de Doença Arterial Coronariana entre estudantes de Enfermagem. Congresso de Iniciação Científica, 12, Mostra de Pesquisa da Pósgraduação, 6, São Paulo: SP, 1601 p.

Brasil (2006). Prevenção clínica de doenças cardiovasculares, cerebrovasculares e renais. Ministério da Saúde, Secretaria de Atenção à Saúde. Brasília.
Barreto S.M., Passos, V.M.A; Giatti, L. (2009). Comportamento saudável entre adultos jovens no Brasil. Revista Saúde Pública, 43 (Supl 2), p. 9-17.

Brandão, I.M.G.; Arcieri, R.M.; Sundefeld, M.L.M.; Moimaz, S.A.S. (2006). Cárie precoce: influência de variáveis sóciocomportamentais e do lócus de controle da saúde em um grupo de crianças de Araraquara, São Paulo, Brasil. Caderno de Saúde Pública, Rio de Janeiro, 22 (6), jun, p. 1247-1256.

Brandão, M.P. et al. (2008). Factores de risco cardiovascular numa população universitária portuguesa [1]. Revista Portuguesa de Cardiologia, 27 (1), p. 7-25. Brandão, M.P.; Pimentel, F.L.; Cardoso, M.F. (2011). Impact of academic exposure on health status of university students. Revista de Saúde Pública, 43 (1), p. 49-58.

Campagnoli, T.; Gonzalez, L.; Cruz, F.S. (2012). Salt intake and blood pressure in the university of Asuncion-Paraguay youths: a preliminary study. Jornal Brasileiro de Nefrologia, 34 (4), p.361-368.

Correia, B.R.; Cavalcante, E.; Santos, E.D.A. (2010). Prevalência de fatores de risco para doenças cardiovasculares em estudantes universitários. Revista da Sociedade Brasileira de Clinica Médica, v.8, p. 25-29.

Dela Coleta, M.F (2010). Crenças sobre comportamentos de saúde e adesão a prevenção e controle de doenças cardiovasculares. Mudanças-Psicologia da Saúde, 18 (1-2), Jan-Dez, p. 69-78.

Diretrizes da Sociedade Brasileira de Cardiologia (2009-2014). Pocket Book. Rio de Janeiro: SBC Tecnologia da Informação e Comunicação, Núcleo Interno de Publicação.

Feliciano-Alfonso et al. (2010).

Cardiovascular risk fators and metabolic syndrome in a population of yong students from the national university of Colombia. Revista da Associação Médica Brasileira, 56 (3), p. 293-298.

Freitas, R.W.J.F. et al. (2013). Análise do 
perfil lipídico de uma população de estudantes universitários. Revista LatinoAmericana de Enfermagem, 21 (5); set-out. Gomes, E.B, Moreira T.M.M.; Pereira, H,C,V.; Sales, I.B., Lima, F.E.T.; Freitas, C.H.A.; Rodrigues, D.P. (2012). Fatores de risco cardiovascular em adultos jovens de um município do Nordeste brasileiro. Revista Brasileira de Enfermagem. Brasília, v. 65, n.4, jul/ago, p. 594-600.

Guerra, A.C.X. (2010). Impacto do Locus de controle e Estratégias de copping em doentes com dor crônica. Dissertação de Mestrado em Psicologia. Universidade Católica Portuguesa, Faculdade de Filosofia de Braga, Rio de Janeiro, Brasil.

Hayasida, N.M.D.A. (2010). Intervenção Cognitivo- Comportamental pré e pós Cirurgia de Revascularização do Miocárdio, em Manaus/AM. Tese de Doutorado apresentada à Faculdade de Filosofia, Ciências e Letras de Ribeirão Preto/USP. Não publicada.

Iser, B.P.M. (2011). Fatores de risco e proteção para doenças crônicas não transmissíveis obtidos por inquérito telefônico - Vigitel Brasil-2009. Revista Brasileira de Epidemiologia, 14 (1), Supl.: p. 90-102.

Joia, L.C. (2010). Perfil do estilo de vida individual entre estudantes universitários. Revista Movimenta. v. 3, N1.

Liparotti, J.R. (2007). Estilo de Vida individual de adultos jovens universitários com excesso de peso da Universidade Católica de Murcia - Espanha. Tese de Doutorado em Ciências da Saúde Universidade Federal do Rio Grande do Norte, Natal, RN.

Madureira, A.S., Corseuil, H.X., Pelegrini, A., Petroski, E.L. (2009) Associação entre estágios de mudança de comportamento relacionados à atividade física e estado nutricional em universitários. Cadernos de Saúde Pública, Rio de janeiro, v. 25, n. 10, out, p. 2139-2146.

Marcondelli, P.; Costa, T.H.M; Schmtz, B.A.S
(2008). Nível de atividade física e hábitos alimentares de universitários do $3^{\circ}$ ao $5^{\circ}$ semestres da área da saúde. Revista de Nutrição, Campinas, v. 21, n.1, jan/fev, p. 39-47.

Mariano, K.G.T.S. et al. (2013). Identificação de fatores de risco para o desenvolvimento de síndrome metabólica e doença cardiovascular em estudantes universitários. Cadernos da Escola de Saúde, Curitiba, v. 2, p. 50-62, ISSN 19847041.

Martins, N.S. (2013). Avaliação dos fatores de risco para doenças cardiovasculares em adolescentes e adultos jovens do Distrito Federal. Trabalho de Conclusão de Curso Universidade de Brasília, Faculdade de Ceilândia.

Martins, M.C.C. et al. (2010). Pressão arterial, excesso de peso e nível de atividade física em estudantes de universidade pública. Arquivos Brasileiros de Cardiologia, 95(2); p. 192-199.

Moreira, T.M.M.; Gomes, E.M; Santos, J.C.S.D. (2010) Fatores de Risco Cardiovasculares em adultos jovens com hipertensão arterial e/ou diabetes mellitus. Revista Gaúcha de Enfermagem. Porto Alegre, v. 31, n. 4, dez, p. 662-669.

Mundim-Masini, A. A. (2009). Fatores de personalidade e percepção de risco podem predizer o comportamento de risco? Um estudo com universitários. $189 \mathrm{f}$. Dissertação de Mestrado em Psicologia Aplicada - Universidade Federal de Uberlândia, Uberlândia, MG.

Organização Mundial de Saúde (OMS). (2014). Organização Pan-Americana da Saúde (OPAS) [homepage na intenet]. Prevenção de doenças crônicas um investimento vital. Recuperado em 03 de março de 2014, de http://www.transdoreso.org/pdf/prevencao_doe nca cronica.pdf.

Petribú, M.M.V.; Cabral, P.C.; Arruda, I.K.G. (2009). Estado nutricional, consumo alimentar e risco cardiovascular: um estudo em universitários. Revista de Nutrição, 22 (6), nov/dez, 837-846. 
Pinheiro, A.F.M.; Fernandes, T.A.B.; Boschetti, L. (2009). Fatores de risco cardiovascular em estagiários e funcionários da clínica-escola da universidade estadual de Goiás. Revista Movimenta, v. 2, n. 4.

Pires, C.G.S. et al. (2013). Prática de atividade física entre estudantes de graduação em enfermagem. Acta Paulista de Enfermagem. 26 (5), p. 436-43.

Simão, C.B.; Nahas, M.V.; Oliveira, E.S.A. (2012). Atividade Física habitual, hábitos alimentares e prevalência de sobrepeso e obesidade em universitários da universidade do planalto catarinense UNIPLAC, LAGES. S.C. Revista Brasileira de Atividade Física \& Saúde.

Timm, L.A., Argimon, I.I.L., Wendt, G.W . (2011). Correlação entre domínios de qualidade de vida e lócus de controle da saúde em idosos residentes na comunidade. Scientia medica. Porto Alegre, v. 21, n. 1, p. 9-13.

Veras, V.S, Monteiro, L.Z., Landim, C.A.P. ; Xavier, A.T.F., Júnior, R.M.M. (2007). Levantamento dos Fatores de Risco para Doenças Crônicas em universitários. Revista Brasileira em Promoção da Saúde. Fortaleza, 20 (3), p. 168-172.

World Health Organization (2012).World health statistics. [S.1.]. 2012. 3(2).
Membro Associada da Sociedade Brasileira de Psicologia Hospitalar - SBPH.

Dados sobre as autoras:

- Isis Gabriela Santos Lemos é mestre em Psicologia (PPGPSI-UFAM). Pesquisadora Colaboradora do Laboratório de Investigação em Ciências Cognitivas (LabICC-FAPSI). Psicóloga Residente em Atenção em Terapia Intensiva- FMT-HVD. Especializanda em Terapia Cognitivo- ComportamentalCognitivo WP.

- Nazaré Maria de Albuquerque Hayasida é doutora em Psicologia (USP-RP). Docente da Universidade Federal do Amazonas/UFAM. Coordenadora do Laboratório de Investigação em Ciências Cognitivas (LabICC-FAPSI)- 